\title{
Identifying Real and Posed Smiles from Observers' Galvanic Skin Response and Blood Volume Pulse
}

\author{
Renshang Gao, Atiqul Islam, Tom Gedeon, and Md Zakir Hossain \\ The Australian National University (ANU), Canberra, Australia \\ \{Renshang.Gao, Atiqul. Islam, Tom.Gedeon, Zakir.Hossain\}@anu.edu.au
}

\begin{abstract}
This study addresses the question whether galvanic skin response (GSR) and blood volume pulse (BVP) of untrained and unaided observers can be used to identify real and posed smiles from different sets of smile videos or smile images. Observers were shown smile face videos / images, either singly or paired, with the intention to recognise each viewed as real or posed smiles. We created four experimental situations, namely single images (SI), single videos (SV), paired images (PI), and paired videos $(\mathrm{PV})$. The GSR and BVP signals were recorded and processed. Our machine learning classifiers reached the highest accuracy of 93.3\%, 87.6\%, 92.0\%, 91.7\% for PV, PI, SV, and SI respectively. Finally, $\mathrm{PV}$ and SI were found to be the easiest and hardest way to identify real and posed smiles respectively. Overall, we demonstrated that observers' subconscious physiological signals (GSR and BVP) are able to identify real and posed smiles at a good accuracy.
\end{abstract}

Keywords: Physiological Signals · Affective Computing · Classification · Machine Learning · Smile.

\section{Introduction}

Philosophers have often referred to the smile as being the reflection of the soul (state of mind), as smiles are one of our most common and easily distinguishable expressions. But the emotions stored in a smile are not just the obvious one. That is, these smiles can be genuine or deceptive. Surprisingly we still know very little about the influence of smiles on others when the smile is deceptive, and people's acceptance or rejection of deceptive smiles as genuine. In different social situations, people may use smiles to cover up their real feelings, disappointment or tension or embarrassment and so on. Some posed smiles are not easily recognized through our eyes. So if we can more accurately identify the meaning of these smiles, it will be of great help in many social situations we (or our AIs or robots) need to face.

Analysis and classification of facial emotions are important research questions in behavioural science. With the advent of computer vision, the literature on facial expressions or emotions is broad, we refer our reader to these literature surveys presented in $[26,13,34]$. Authors in [32] attempted to develop 
more reliable smile detection in realistic situations with the advent of machine learning. This type of smile detector is usually embedded in commercial digital cameras. They only can detect the expression of a smile without identifying whether the smile is genuine or posed. However, classifying real and posed smile through the application of machine learning is comparatively a recent research question. Different researchers have tried different approaches. Eyelid movement has been used as a classifying factor for differentiating between spontaneous and posed smiles [7]. Authors have reached up to 92 percent accuracy for posed smile detection with neural networks. Authors in [2] utilized machine learning on subjects' physiological signals while watching emotionally evocative films to distinguish between different emotions that arose within the subject. Real and posed smiles have been classified from observer's physiological signals in [16]. The authors have used observers' galvanic skin response and blood volume pulse along with pupillary responses. From twenty videos of a benchmark database with twenty four subjects using neural network classification, the authors have reached around 93 percent accuracy.

This study focuses on the research and analysis of subjects' physiological signals. Through processing of the physiological signals generated by observing posed and real smiles, feature extraction, and training classification, create a high-precision classifier to improve the accuracy of predicting real and posed smiles, thereby helping people to more accurately judge human smiles. People are surprisingly bad at differentiating posed and real smiles. In this paper, we have analyzed the accuracy of the proposed classifier, and investigated which training method has higher efficiency in differentiating between real and posed smiles. Also, we analyse feature distributions to find the reasons behind low performances. First, by comparing the accuracy of different individual features, low-precision features are excluded, and high-precision multiple features are used to train a new classifier jointly. The results show that if only low-precision features are excluded, this does not improve the accuracy of the final classifier very well. In the second part of the study, to investigate the distribution of features, and to experiment with independent parameters in the feature list, the experiment found that the final accuracy has been greatly improved. The highest accuracy classifier performs data prediction, and finally shows that the pair data has better predictive power, with an accuracy of 93.3 percent, while the single data achieved up to 92 percent.

\section{Background}

Emotions are one of the essential factors in our lives. Positive emotions can help people maintain a healthy and high quality of life. Negative emotions often bring negative life conditions and even affect physical health. For example, long-term depression, claustrophobia, or irritability may transform into mental illness. If it is not controlled in time, serious life-threatening situations such as injury or suicide may occur! Therefore, emotion recognition has been widely researched in many Fields, such as safe driving, psychological disease monitoring, social safety 
and many more. The extensive state-of-the-art literature surveys in the last few decades is an indication of the wide range of research that is happening in the field of emotion recognition from speech, audiovisual, facial, smile etc. $[11,33$, $22,27]$.

However, it is difficult to judge human emotion changes based only on human body movements or facial expressions, because people often cover their true emotions in different ways, such as smiling [10]. Therefore, the discovery of physiological signals is an important help for emotion recognition. Because when the human body has different emotional changes, physiological signals will have different changes, and generally these changes are spontaneous and not controllable. Therefore, it can reflect the true human emotions without being covered up, helping us to identify human emotion changes more easily. Being one of the emotional cues, a considerable amount of research has been done on the smile. That research can be grouped into a few categories such as smiles in consumer research [31], smile detection [32] and physiological responses during a smile [9]. A significant amount of study suggests that smiles are influential social forces that positively impact interpersonal judgments in many ways. For example, researchers found that people who express genuine smiles are perceived to be more polite, carefree and pleasant $[5,6,23]$. The substantial amount of evidence supporting the social benefits of smiles implies that a smile always convey some form of information. Indeed, researchers believed that people sometimes intentionally strengthen emotional displays to get favourable interpersonal feedback [25]. For instance, service workers often exaggerate their positive emotional displays in order to increase consumer experiences [3]. Smiles can therefore be considered as an important facial capacity in successful social communication. Thus it is important to recognize real and posed smiles not only in human to human communication but also in human machine communication. In this paper, real and posed smiles have been classified from observers' physiological signals using machine learning algorithms.

\subsection{Physiological Signals}

Users' emotions including smile can be recognized in a myriad of ways like facial expression, body pose, audiovisual, gesture recognition and many more but physiological signals are the most useful in the field of human-computer interaction because they cannot be controlled intentionally. There are many physiological signals that can be collected from the human body to get information about internal behaviour and mental states. The main physiological signals include cardiac function, temperature, muscle electrical activity, respiration, electrical activity of the skin and brain, blood volume pulse and so on. This paper will mainly focus on the analysis of two physiological signals: skin conductance and blood volume pulse.

GSR (galvanic skin resistance), a general term for the galvanic activity or EDA (electrodermal activity) refers to changes in sweat gland activity, reflecting the intensity of our emotional state, also known as emotional arousal [4]. Our level of emotional arousal changes depending on the environment if something is 
frightening, threatening, happy or otherwise related to emotions, then our subsequent emotional response changes also increase endocrine sweat gland activity. Studies have shown that this is related to emotional arousal. It is worth noting that both positive ("joyful" or "happy") and negative ("threat" or "frightening") stimuli can cause an increase in arousal and lead to an increase in skin response. Therefore, the GSR signal does not represent the type of emotion, but rather its intensity. An extensive review on literature shows that GSR has been very popular in the last two decades for working with emotion or smiles $[20,8$, 28].

Photoplethysmography (PPG) allows infrared light to pass through the tissue and measure the absorption of light by blood flowing through the blood vessels below the skin. It can be used to detect the blood flow rate of the heartbeat, also known as blood volume pulse (BVP). BVP is not only a convenient measurement of heart rate variability but also a valuable information source for emotion recognition. The BVP signal indicates the changes in blood volume and blood flow through the human body [14]. Many researchers include heart rate or BVP in their experiment or database of psycho-physiological classification or recognition [21].

\section{$3 \quad$ Experimental Methodology}

The aim of our study is to analyze the physiological signal data produced by feeling as evoked by watching real and posed smiles. After finding a suitable classification method, we have identified real and posed smiles from observers' physiological signals to demonstrate observers' emotional communication.

\subsection{Smile Videos and Images Stimuli}

The smiles stimuli were collected from the UvA-NEMO dataset [15]. A total of 60 videos were randomly selected for this experiment. We created four categories out of these 60 videos, namely PV (paired videos), PI (paired images), SV (single video), and SI (single image). When the same person was viewed by observers in both real and posed smile videos, we report this as "paired videos", otherwise we use the term "single video". A similar rule was applied for the images we used. Here, image means a single frame and video means a sequence of frames. The selected videos/images were processed using oval masks [17] to keep the face portion only, and presented to the observer in an order balanced way to avoid any order effects. We kept the length of each video the same as the source video in the dataset. The length of the videos usually spans from a minimum of 2 seconds to a maximum of 8 seconds. In case of images, we chose one of the middle frames from each video and showed the frame for five seconds. Overall, we considered 20 videos for PV, 10 videos for SV, 20 images for PI, and 10 images for SI respectively. 


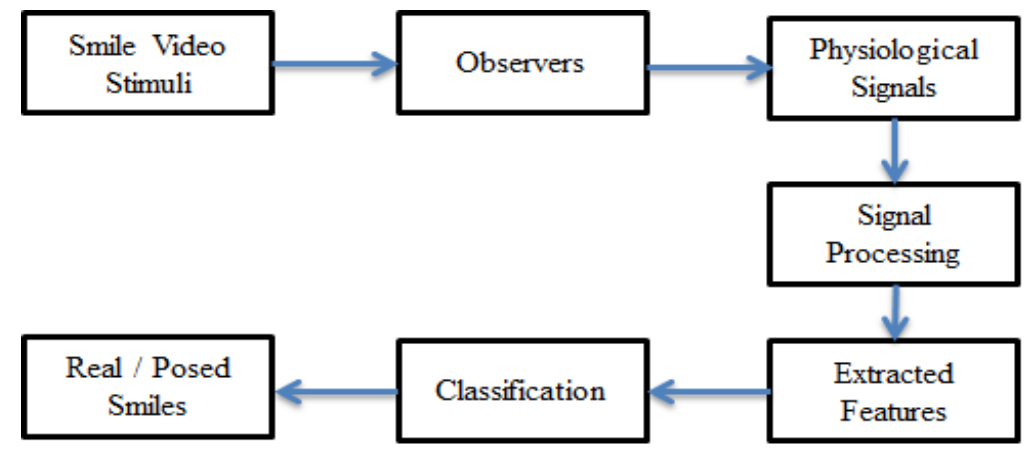

Fig. 1. Experimental Procedure.

\subsection{Participants/Observers}

We invited 25 participants (also called observers as they watch or observe visual stimuli) who were the university students and volunteered to take part in the experiment. Amongst the participants, we had 13 males and 12 females with age range $24.6 \pm 2.54$ (average \pm standard deviation). All the participants had normal or corrected to normal vision and provided written consent prior to their participation. Approval from our University's Human Research Ethics Committee was received before the experiment.

\subsection{Physiological Data Acquisition}

In order to detect real smiles from mixed presentations of real and posed smiles, we recorded galvanic skin response (GSR) and blood volume pulse (BVP) using an Empatica E4 [12] on the wrist of each observers' non-dominant arm. The GSR and BVP data were recorded at a sampling rate of $4 \mathrm{~Hz}$ and $64 \mathrm{~Hz}$ respectively.

\subsection{Experimental Procedure}

Observers were briefed about the experiment after arrival at the laboratory, and asked to sign the consent form. They were seated on a static chair, facing a 15.6 inch ASUS laptop in a sound-attenuated, dimly lit, closed room. Sensors were attached to measure their GSR and BVP signals. Their chairs were moved forward or backwards to adjust the distance between the chair and laptop. Observers were asked to limit their body movements in order to reduce undesired artefacts in the signals. The smile videos or images were presented to the observers in a randomised fashion considering each experiment separately (i.e SI, SV, PI, and PV). At the end of each (or pair) video or image, the observer was asked to choose whether the smile was real or posed by answering the question on the laptop into a web interface. The total duration of the experiment was around 45 minutes. After completing the experiment, the sensors were removed, and the observers were thanked for their participation. Overall, our experimental 
procedure is shown in Fig. 1. We have stored the experimental data for future post processing. Filtering and normalization were applied on each signal. After that we have extracted several features from each signal. Then the classification algorithm was applied on the extracted features and smiles are identified as real or posed.

\subsection{Signal Processing}

Generally, the recorded signals are affected by noise due to small signal fluctuations and observers' movements during the experiment. So we employed median filters to reduce the effect of noise: a one-dimensional median filter, with parameter use of $20^{\text {th }}$-order. Finally to overcome the individual human differences on the data, we normalise the data into the range of $\left[\begin{array}{ll}0 & 1\end{array}\right]$. The normalization technique used here is Linear Scaling, which is obtained by calculating the maximum and minimum values as shown in equation 1 . Here $x_{i}$ is the physiological signal vector.

$$
\text { Normalization }=\frac{x_{i}-\min \left(x_{i}\right)}{\max \left(x_{i}\right)-\min \left(x_{i}\right)}
$$

In this experiment, we used Matlab for signal processing. The signal noise has been reduced by removing all the outliers after filtering. There are four sets of physiological signals (SI, SV, PI and PV). So after normalizing the data, we can compare those different categories of data as they are normalised to the same levels by observer.

\subsection{Feature Extraction}

After the normalization and filtering, a number of features have been calculated from the filtered and normalized signals. We extracted several features from each video/image for each observer's physiological signals. The features have been selected based on the state-of-the-art literature in human emotion, physiological signal and affective computing $[24,1,19]$. Initially 20 features have been extracted from each physiological signal. The features set includes average, root mean square (RMS), variance, standard deviation, Hjorth mobility, sum, simple square integral (SSI), approximate Entropy (ApEn), mean absolute deviation (MAD), Kurtosis, sample Entropy, Shannon Entropy, peak to peak, root-sumof-squares (Rssq), mean frequency, occupied bandwidth, equivalent noise bandwidth, band power, Fuzzy Entropy and detrended fluctuation analysis (DFA). DFA determines the self similarity level of physiological signals. We extracted a sample for each of the experimental condition such as SI, SV, PI, PV from each participant. A total of 20 features have been extracted from each sample of a participant for each physiological signal. So, from each participant 160 features $(4$ samples $($ SI, SV, PI and PV) $\times 20$ Features $\times 2$ Physiological signals (GSR, BVP)) have been extracted. For each condition the real and posed smiles are balanced; for example, condition SI got 5 real smile images and 5 posed smile images. 


\section{Experimental Results and Discussion}

The data is analysed according to four groups, namely SI, SV, PI, and PV as mentioned earlier. The four sets of data were classified separately using normalized features. After initial training the prediction accuracy of all the data were quite low as shown in Table 1. In the table, we have shown the classification method that achieve the maximum accuracy amongst the methods we used. As an example, we used all four methods (Fine KNN, Kernel Naive Bayes, Coarse Tree Logistic Regression) for PV, but we achieved maximum accuracy with Coarse Tree. We can see that the highest accuracy is $62.5 \%$ for PV, which is quite low. Therefore, we focus on two factors: the type of features selected by the classifier, and the number of features for training the classifier.

Table 1. Initial accuracy of classification using all the normalized features

\begin{tabular}{lll}
\hline Type of Data Accuracy & Method of classification \\
\hline SI & $53.6 \%$ & Fine KNN \\
SV & $58.6 \%$ & Kernel Naive Bayes \\
PV & $62.5 \%$ & Coarse Tree \\
PI & $57.0 \%$ & Logistic Regression \\
\hline
\end{tabular}

\subsection{Analysis of the Feature Performance}

As a first approach, we re-analyzed each of the features to find the features' performance. It is impossible to judge the accuracy by comparing the classification methods of different categories, rather we need to compare the accuracy of the same classification method / condition. There are four main types of classification methods with good results: Coarse Tree, Logistic regression, Fine KNN, and Kernel Naive Bayes. Therefore, we computed the accuracy for each situations (i.e. SI, PI, SV, and PV) separately considering each classification methods where we achieved the highest accuracies, i.e Fine KNN for SI, Logistic Regression for PI, and so on.

First, we trained all of the features and recorded the accuracy. After excluding some features with low accuracy and using the features with the highest accuracy, we found that removing some features can improve accuracy. We have selected 10 high accuracy features based on their individual performance. The selected features set includes average, root mean square (RMS), variance, standard deviation, Hjorth mobility, approximate Entropy (ApEn), mean absolute deviation (MAD), Kurtosis and mean frequency. Some of these results indicate that the accuracy of a single feature can be slightly higher than a classifier that used multiple features for training. The increased range is about 2.3 percent to 6 percent as shown in Table 2. So we can conclude that removing some low accuracy features is not the main reason for improving accuracy. 
Table 2. After feature selection accuracy has increased

\begin{tabular}{llll}
\hline $\begin{array}{l}\text { Type of Accuracy with Accuracy after } \\
\text { Data }\end{array}$ & $\begin{array}{l}\text { Increase } \\
\text { all features }\end{array}$ & feature selection range \\
\hline SI & $53.6 \%$ & $59.5 \%$ & $+5.9 \%$ \\
SV & $58.6 \%$ & $60.9 \%$ & $+2.3 \%$ \\
PV & $62.5 \%$ & $65.5 \%$ & $+3.0 \%$ \\
PI & $57.0 \%$ & $61.1 \%$ & $+4.1 \%$ \\
\hline
\end{tabular}

\subsection{Feature Distributions}

To examine the reason of a feature with respect to another feature, we checked the distribution of features with trained and untrained data as shown in Fig. 2 as an example considering PV. By observation, we can see that the posed smile data shows a lot of loss during training as displayed in Fig. 2 (b), and the real smile data is alright during training as shown in Fig. 2 (d). In other words,

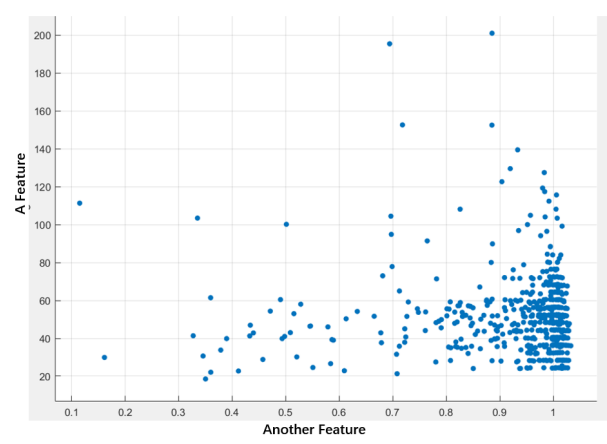

((a)) Posed smile untrained data

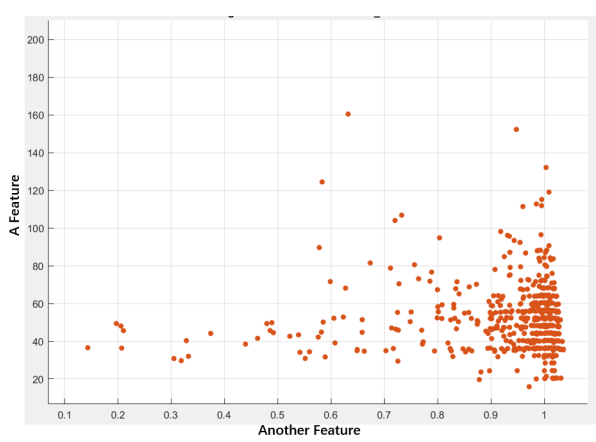

((c)) Real smile untrained data

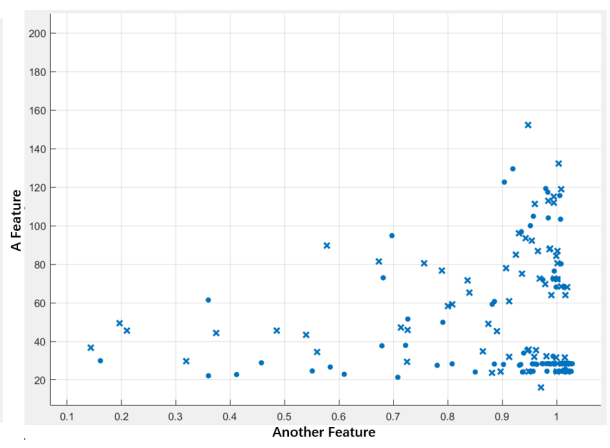

((b)) Posed smile trained data

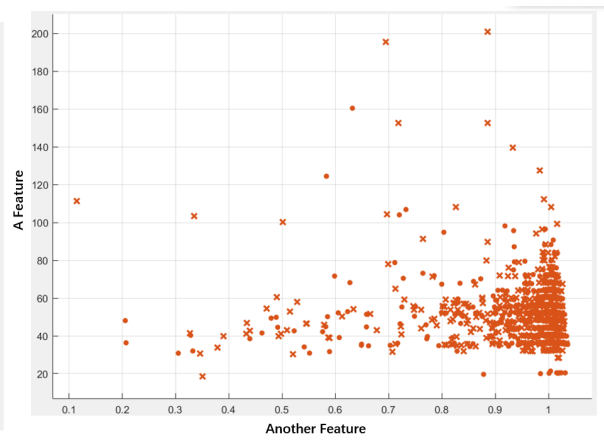

((d)) Real smile trained data

Fig. 2. Features distribution before and after classification for real and posed smiles 
the classifier erroneously assigns posed smile data to real smiles data during the classification process. Therefore, in the entire training set, the data assigned to real smiles set also includes the data of some posed smiles. It is because posed smiles take longer time to generate as well as recognise compared to the real smiles $[7,16]$.

Due to the uneven features distribution, the classification performances are also different considering the real and posed smiles separately. For instance, our classifier achieves $90 \%$ and $41 \%$ correctness for real and posed smiles separately for PV (i.e $65.5 \%$ on average as shown in Table 2). It is because the classifier chose the 30 percent test data randomly and unevenly, which did not match with training data. For this reason, a few posed smile features were considered as real smile features. To overcome this lagging, we applied leave-one-participant-out method (the preferred method when using human sensor data [30,29]) to improve the accuracies. The leave-one-out approach was applied to validate the performance, which used each subject's data as testing set while others as training set. Therefore the validation ran a total of 25 loops, and the result was the average of each loop. The leave-one-out validation process was repeated for 10 times and we average the results to get the final result. We used k-nearest neighbour (KNN), Naive Bayes, Decision Tree and Logistic Regression classifiers. The performance parameters were: 1 nearest neighbours, kernel function, Coarse function and logistic function respectively.

\subsection{Predictive Data Testing}

According to the above analysis of features, the number of features is not the main reason for improving accuracy, and now the classifier with the highest accuracy is selected for predictive data testing. The test method removes one observer's data for testing and remaining 24 observers' data to train the classifier, thus full-filling the requirements for the leave-one-participant-out method. The Table 3 records the accuracy of the predictions for GSR and BVP signals. It is important to note that, we illustrated the results from first three test par-

Table 3. Classification Accuracies (in \%) of Three Participants (as an example)

\begin{tabular}{ccccccc}
\hline \multirow{2}{*}{ Conditions } & \multicolumn{4}{c}{ GSR } & \multicolumn{4}{c}{ BVP } \\
\cline { 2 - 7 } & Participant & Participant & Participant & Participant & Participant & Participant \\
& P01 & P02 & P03 & P01 & P02 & P03 \\
\hline SI & 78.6 & 79.0 & 79.0 & 92.3 & 91.1 & 92.3 \\
SV & 81.1 & 82.9 & 80.9 & 91.3 & 89.8 & 94.5 \\
PV & 90.0 & 88.1 & 89.5 & 94.8 & 91.1 & 95.2 \\
PI & 88.5 & 87.7 & 87.1 & 58.8 & 65.4 & 65.5 \\
\hline
\end{tabular}

ticipants (P01, P02, and P03) as an example as shown in Table 3, because the classifiers achieve similar results for all test participants. Average accuracy of all 


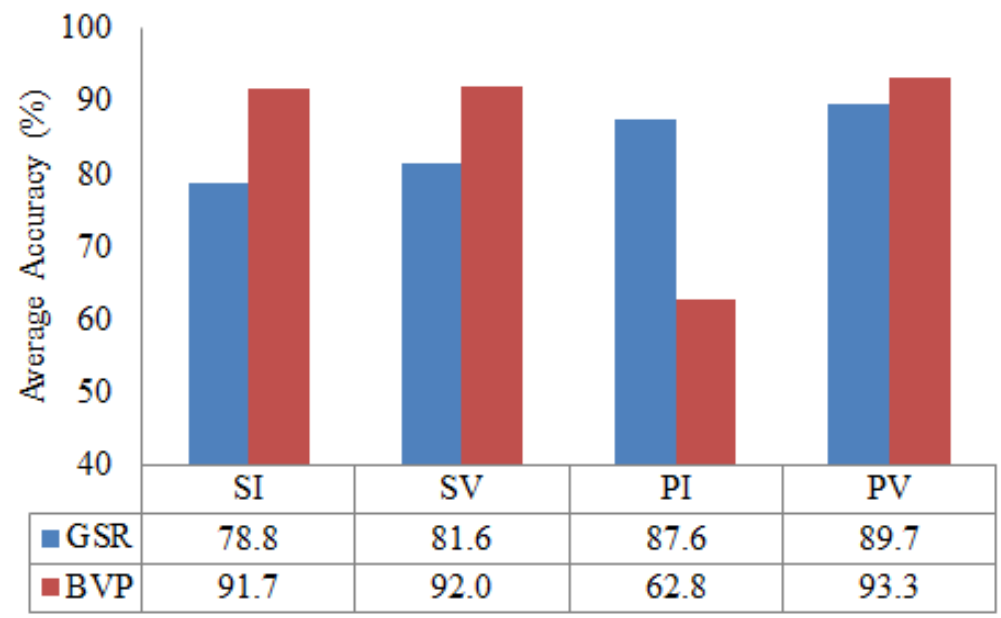

Fig. 3. Overall accuracy of classification for all participants for both GSR and BVP signals.

25 participants is shown in Fig. 3. It can be seen from Fig. 3 that highest accuracies are recorded from BVP data considering all situations except PI. This is likely to be because the PI data were noisy and corrupted for most of the observers for this case. For all other cases, it can be seen that highest accuracies were found from PV followed by PI, SV, and SI respectively. BVP was found to be more reliable compared to GSR in this case.

\section{Conclusions}

In this study, we have computed the accuracy of classifiers when differentiating between real and posed smile from participants' GSR and BVP data. An exclusion method was used to remove low-efficiency features, leaving high-precision features for training the classifiers. The results showed that this method does not improve the accuracy very well. In subsequent experiments, the classifier was trained using the leave-one-participant-out approach. We can see that the results have greatly improved for all four situations namely SI, SV, PI, and PV. After overall analysis, it was shown that in condition PV it is most easy to recognise genuine smiles and differentiate them from posed smiles (accuracy 93.3\%) followed by PI, SV, and SI. In other words, paired videos / images are easy compared to the single videos / images, and videos are easier to recognise compared to single images. This demonstrates experimentally a general hypothesis of human intuition, which tells us about the applicability of AI techniques for human facial expression recognition from only looking at (or observing) them without connecting any devices to the person displaying the emotion. In our future research we will include more observers from diversified social groups and 
age ranges. We will also include other state-of-the-art databases, diverse features, and well-known feature selection methods $[8,18,33,34]$.

\section{References}

1. Ayata, D., Yaslan, Y., Kamaşak, M.: Emotion recognition via galvanic skin response: Comparison of machine learning algorithms and feature extraction methods. Istanbul University-Journal of Electrical \& Electronics Engineering 17(1), 3147-3156 (2017)

2. Bailenson, J.N., Pontikakis, E.D., Mauss, I.B., Gross, J.J., Jabon, M.E., Hutcherson, C.A., Nass, C., John, O.: Real-time classification of evoked emotions using facial feature tracking and physiological responses. International journal of humancomputer studies 66(5), 303-317 (2008)

3. Barger, P.B., Grandey, A.A.: Service with a smile and encounter satisfaction: Emotional contagion and appraisal mechanisms. Academy of management journal 49(6), 1229-1238 (2006)

4. Braithwaite, J.J., Watson, D.G., Jones, R., Rowe, M.: A guide for analysing electrodermal activity (eda) \& skin conductance responses (scrs) for psychological experiments. Psychophysiology 49(1), 1017-1034 (2013)

5. Bugental, D.B.: Unmasking the" polite smile" situational and personal determinants of managed affect in adult-child interaction. Personality and Social Psychology Bulletin 12(1), 7-16 (1986)

6. Deutsch, F.M., LeBaron, D., Fryer, M.M.: What is in a smile? Psychology of Women Quarterly 11(3), 341-352 (1987)

7. Dibeklioglu, H., Valenti, R., Salah, A.A., Gevers, T.: Eyes do not lie: Spontaneous versus posed smiles. In: Proceedings of the 18th ACM international conference on Multimedia. pp. 703-706 (2010)

8. Dzedzickis, A., Kaklauskas, A., Bucinskas, V.: Human emotion recognition: Review of sensors and methods. Sensors 20(3), 592 (2020)

9. Ekman, P., Davidson, R.J.: Voluntary smiling changes regional brain activity. Psychological Science 4(5), 342-345 (1993)

10. Ekman, P., Friesen, W.V.: Felt, false, and miserable smiles. Journal of nonverbal behavior 6(4), 238-252 (1982)

11. El Ayadi, M., Kamel, M.S., Karray, F.: Survey on speech emotion recognition: Features, classification schemes, and databases. Pattern Recognition 44(3), 572587 (2011)

12. a medical-grade wearable device that offers real-time physiological data acquisition (2020), https://www.empatica.com/en-int/research/e4/

13. Fasel, B., Luettin, J.: Automatic facial expression analysis: a survey. Pattern recognition 36(1), 259-275 (2003)

14. Gouizi, K., Bereksi Reguig, F., Maaoui, C.: Emotion recognition from physiological signals. Journal of medical engineering \& technology 35(6-7), 300-307 (2011)

15. H.Dibeklioglu, A.A.Salah, T.: Recognition of genuine smiles. IEEE trans Multimed(17), pp.279-294 (2015)

16. Hossain, M.Z., Gedeon, T.: Classifying posed and real smiles from observers' peripheral physiology. In: Proceedings of the 11th EAI International Conference on Pervasive Computing Technologies for Healthcare. pp. 460-463 (2017)

17. Hossain, M.Z., Gedeon, T.: Discriminating real and posed smiles:human and avatar smiles. Tech. rep., Brisbane, QLD, Australia (November 2017) 
18. Hossain, M.Z., Kabir, M.M., Shahjahan, M.: A robust feature selection system with colin's cca network. Neurocomputing 173, 855 - 863 (2016)

19. Islam, A., Ma, J., Gedeon, T., Hossain, M.Z., Liu, Y.H.: Measuring user responses to driving simulators: A galvanic skin response based study. In: 2019 IEEE International Conference on Artificial Intelligence and Virtual Reality (AIVR). pp. 33-337. IEEE (2019)

20. Jerritta, S., Murugappan, M., Nagarajan, R., Wan, K.: Physiological signals based human emotion recognition: a review. In: 2011 IEEE 7th International Colloquium on Signal Processing and its Applications. pp. 410-415. IEEE (2011)

21. Koelstra, S., Muhl, C., Soleymani, M., Lee, J.S., Yazdani, A., Ebrahimi, T., Pun, T., Nijholt, A., Patras, I.: Deap: A database for emotion analysis; using physiological signals. IEEE transactions on affective computing 3(1), 18-31 (2011)

22. Mehta, D., Siddiqui, M.F.H., Javaid, A.Y.: Facial emotion recognition: A survey and real-world user experiences in mixed reality. Sensors 18(2), 416 (2018)

23. Mueser, K.T., Grau, B.W., Sussman, S., Rosen, A.J.: You're only as pretty as you feel: facial expression as a determinant of physical attractiveness. Journal of Personality and Social Psychology 46(2), 469 (1984)

24. Picard, R.W., Vyzas, E., Healey, J.: Toward machine emotional intelligence: Analysis of affective physiological state. IEEE transactions on pattern analysis and machine intelligence 23(10), 1175-1191 (2001)

25. Pugh, S.D.: Service with a smile: Emotional contagion in the service encounter. Academy of management journal 44(5), 1018-1027 (2001)

26. Samal, A., Iyengar, P.A.: Automatic recognition and analysis of human faces and facial expressions: A survey. Pattern recognition 25(1), 65-77 (1992)

27. Sebe, N., Cohen, I., Gevers, T., Huang, T.S.: Multimodal approaches for emotion recognition: a survey. In: Internet Imaging VI. vol. 5670, pp. 56-67. International Society for Optics and Photonics (2005)

28. Shu, L., Xie, J., Yang, M., Li, Z., Li, Z., Liao, D., Xu, X., Yang, X.: A review of emotion recognition using physiological signals. Sensors 18(7), 2074 (2018)

29. Song, T., Lu, G., Yan, J.: Emotion recognition based on physiological signals using convolution neural networks. In: Proceedings of the 202012 th International Conference on Machine Learning and Computing. pp. 161-165 (2020)

30. Teichmann, D., Klopp, J., Hallmann, A., Schuett, K., Wolfart, S., Teichmann, M.: Detection of acute periodontal pain from physiological signals. Physiological measurement 39(9), 095007 (2018)

31. Wang, Z., Mao, H., Li, Y.J., Liu, F.: Smile big or not? effects of smile intensity on perceptions of warmth and competence. Journal of Consumer Research 43(5), 787-805 (2017)

32. Whitehill, J., Littlewort, G., Fasel, I., Bartlett, M., Movellan, J.: Toward practical smile detection. IEEE transactions on pattern analysis and machine intelligence 31(11), 2106-2111 (2009)

33. Wu, C.H., Lin, J.C., Wei, W.L.: Survey on audiovisual emotion recognition: databases, features, and data fusion strategies. APSIPA transactions on signal and information processing $\mathbf{3}$ (2014)

34. Zeng, Z., Pantic, M., Roisman, G.I., Huang, T.S.: A survey of affect recognition methods: Audio, visual, and spontaneous expressions. IEEE transactions on pattern analysis and machine intelligence 31(1), 39-58 (2008) 